\title{
Occasional reviews
}

\section{Current role of positron emission tomography in thoracic oncology}

\author{
Val J Lowe, Keith S Naunheim
}

Positron emission tomography (PET) has been in existence and continuously developing over the past 20 years. In the last few years steady advances in the technology used for PET and complementary progress in supporting computer equipment have led to the ability to perform whole body imaging with this modality. With this evolution has come an improved capability for the diagnosis, staging, and post-treatment surveillance of malignancies in the thoracic cavity. This report will review the current status of PET imaging for the investigation of thoracic oncology.

\section{PET scan technique}

PET imaging involves the use of specialised radiation sensitive cameras which detect specific radioactive isotopes that decay by positron emission. Most of the common molecules involved in organic processes can be labelled with positron emitting isotopes. Atoms of low atomic number such as carbon, oxygen, nitrogen, and fluorine have positron emitting isotopes. Molecules of specific functional import, labelled with positron emitting isotopes, can be injected intravenously into patients and an image of their distribution can be obtained using a PET camera. Although many different tracers can be manufactured and have been utilised to evaluate malignancy, this review will focus on the use of one tracer, ${ }^{18} \mathrm{~F}$-fluorodeoxyglucose (FDG), which is the most widely used for detection of malignancy.

Malignant tumours usually demonstrate rapid cell proliferation with a proportionally increased cellular metabolism. Physiologically, this translates into increased glucose metabolism in malignant cells, a finding first reported in the 1930s. ${ }^{1-3}$ Comparable enhancements of glucose and FDG uptake in malignant cells have permitted the identification of malignancy using PET imaging. ${ }^{4} \mathrm{~A}$ unique feature of FDG as a marker of glucose metabolism is the fact that, after FDG phosphorylation, FDG-6- $\mathrm{PO}_{4}$ does not proceed further in the metabolic pathway but remains trapped within tumour cells, thus becoming a marker of metabolism. This persistence of the tracer in the tumour is essential for successful imaging to be performed. $^{5}$ The relative uptake of FDG can be used as an indicator of tumour aggressiveness and correlates with tumour growth rates. ${ }^{6}$

PET imaging provides numerical data by quantitating the number of positron emissions.
These data accurately reflect the amount of FDG accumulating in a selected region. The standardised uptake ratio (SUR) is an uptake value normalised for patient body weight and imaging dose that provides a means of comparison of FDG uptake between patients. It is calculated in the following way:

$\mathrm{SUR}=\frac{\text { mean selected region activity }(\mathrm{mCi} / \mathrm{ml})}{\text { injected dose }(\mathrm{mCi}) / \text { body } \mathrm{wt}(\mathrm{g})}$

SUR values of $>2.5$ have been considered indicative of malignancy by some authors while slightly different values are used by others. Still other investigators depend instead on visual interpretation of abnormality-that is, FDG uptake greater than mediastinal uptake-as being abnormal. FDG imaging is performed in the fasting state to minimise competitive inhibition of FDG uptake by glucose. The effect of diabetes and serum hyperglycaemia on the uptake of FDG in primary lung cancers has not been proven to affect the accuracy of the test although there is a concern because elevated serum glucose levels can result in decreased FDG accumulation in tumour. ${ }^{7}$ Other PET tracers are available that could avoid this problem if using FDG in hyperglycaemic patients is found to result in a reduction of diagnostic accuracy. ${ }^{8}$ The PET scans are performed 50-60 minutes after intravenous administration of 10.0-20.0 mCi of FDG and are usually completed after an additional hour of tomography. ${ }^{9}$ Images are generally displayed as axial, coronal, and sagittal views as well as three-dimensional surface projections. Semiquantitative assessment, as described above, of particular lesions is also often performed. Although images can be obtained of localised areas, the same injection will allow whole body images to be obtained. While images of localised areas may only require 15-30 minutes, a whole body image usually requires an hour or more. In the near future whole body images may well be performed in about 30-40 minutes, largely due to new imaging software and faster computers. Development of new kinds of light detection crystals for use in the next generation of PET scanners is also underway and this may allow improved image resolution when compared with today's systems.

Evaluation of focal lung abnormalities Focal lung abnormalities such as a defined nodule or a non-specific opacity often need to be evaluated for malignancy. Imaging with 
chest radiography, computed tomography (CT) or magnetic resonance imaging (MRI) will rarely characterise focal lung abnormalities definitively as benign or malignant since the classic radiographic finding of centralised calcification is only infrequently seen. ${ }^{10}{ }^{11}$ Several invasive modalities are available to assist in the diagnosis of these radiographic lesions. Bronchoscopy, including bronchial washings and brushing, has a limited sensitivity of $65 \%$ for malignancy and the addition of transbronchial biopsy only increases the sensitivity to $79 \%{ }^{12}{ }^{13}$ Bronchoscopy proves most helpful in the more central lesions that are bronchoscopically accessible. Peripheral lesions can also be biopsied percutaneously with the help of CT guidance. Published reports suggest that CT guided transthoracic needle aspiration (TTNA) has a sensitivity and specificity of $94-98 \%$ and $91-96 \%$, respectively. ${ }^{14} 15$

Negative results from transbronchial biopsies and/or transthoracic needle aspirations (TTNA) can not be accepted as true negatives at all times. For example, in one study in which TTNA of discrete pulmonary lesions was evaluated in 181 patients, $40 \%$ of the biopsy specimens failed to produce a definitive diagnosis and $40 \%$ of this subset subsequently proved to be malignant lesions. ${ }^{16}$ A definitively negative diagnosis by percutaneous fine needle biopsy could be established in only $16 \%$ of the patients with benign conditions in this study. In another report the histological reports were reviewed following diagnostic thoracotomy performed on patients in whom TTNA was considered difficult, inappropriate, or was unsuccessfully attempted. Up to $65 \%$ of these nodules were incorrectly characterised prior to surgery, and in $50 \%$ of patients found to have cancer, malignancy was not the preoperative diagnosis. ${ }^{17}$ There are also risks to these more aggressive diagnostic manoeuvres. Percutaneous biopsy has an $18-26 \%$ risk of pneumothorax and $10-15 \%$ of patients with postprocedure pneumothorax require chest tubes. $^{15} 18$

While the recently popularised technique of thoracoscopic lung biopsy provides a definitive diagnosis, in a recent series over $50 \%$ of radiographically indeterminate nodules resected by thoracoscopy were benign. ${ }^{19}$ Thus, half of this patient population underwent an invasive and potentially avoidable surgical procedure.

An accurate non-invasive test for evaluating indeterminate pulmonary lesions could potentially avoid considerable patient morbidity and potentially reduce lower medical costs by obviating invasive procedures. Recently published data have demonstrated the ability of PET to characterise lung abnormalities and have been particularly encouraging.

After the identification of a pulmonary abnormality by an anatomical study such as a chest radiograph, FDG-PET imaging can be performed to evaluate the metabolic activity of the lesion in an attempt to distinguish a benign from a malignant process. Investigators have used FDG-PET in the assessment of focal pulmonary nodules and other pulmonary opaci-
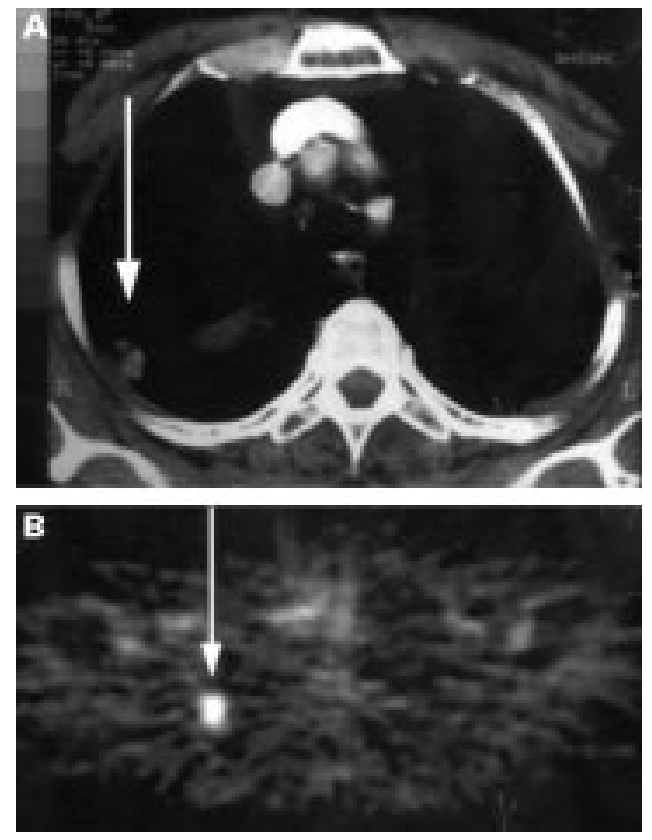

Figure 1 Pulmonary nodule (arrow) in right upper lobe on $(A) C T$ scan that is hypermetabolic (arrow) on (B) FDG-PET scan. A biopsy specimen was taken of the nodule and was found to be adenocarcinoma.

ties. In an early report one centre investigated FDG-PET imaging of solitary pulmonary nodules in 30 patients and found that PET had a sensitivity and a specificity of $95 \%$ and $80 \%$, respectively. ${ }^{20}$ In another report 51 patients with focal opacities that could not be characterised as benign or malignant by chest radiography or CT scanning underwent a PET scan. The sensitivity and specificity of FDG-PET imaging for malignancy were found to be $100 \%$ and $89 \%$, respectively. ${ }^{21}$ When FDG uptake within the focal opacities was analysed a highly statistically significant difference was found between the PET SUR value of malignant and benign lesions $(p=0.0001)$. Figure 1 shows a case of an indeterminate pulmonary nodule that was correctly characterised as tumour by PET imaging.

Table 1 lists a review of data evaluating the use of PET imaging in pulmonary abnormalities. This list includes the most recent comprehensive reports from the institutions reporting PET assessments of lung abnormalities. ${ }^{22-28}$ Some investigators have included only solitary pulmonary nodules while others have widened the criteria to include any suspicious opacity. The data show that PET imaging performs equally well in either group with an average sensitivity and specificity of $95 \%$ and $88 \%$ for the detection of malignancy.

As with most medical tests, PET imaging is not $100 \%$ accurate. The data demonstrate that there will be a small proportion of pulmonary opacities that will be metabolically active yet benign, resulting in false positive PET scans (table 2). Some active infectious or inflammatory lesions may have significant uptake of FDG. Tuberculous granulomas, coccidiomycosis, aspergillosis and histoplasmosis were causes of some of the false positive results seen in the studies listed. However, these false 
Table 1 FDG PET studies of pulmonary opacities or solitary pulmonary nodules

\begin{tabular}{|c|c|c|c|c|}
\hline \multirow{2}{*}{$\begin{array}{l}\text { Reference } \\
\text { Bury }(1996)^{22}\end{array}$} & \multicolumn{2}{|c|}{$\begin{array}{l}\text { Patients } \\
\text { (malignant/benign) }\end{array}$} & \multirow{2}{*}{$\begin{array}{l}\text { Sensitivity } \\
100 \%\end{array}$} & \multirow{2}{*}{$\begin{array}{l}\text { Specificity } \\
88 \%\end{array}$} \\
\hline & $33 / 17$ & & & \\
\hline Duhaylongsod $(1995)^{23}$ & $59 / 28$ & - & $97 \%$ & $81 \%$ \\
\hline Gupta $(1996)^{24}$ & $45 / 16$ & & $93 \%$ & $88 \%$ \\
\hline Knight $(1996)^{25}$ & $32 / 16$ & & $100 \%$ & $63 \%$ \\
\hline Kubota $(1990)^{26}$ & $12 / 10$ & - & $83 \%$ & $90 \%$ \\
\hline Lowe $(1997)^{27}$ & $120 / 77$ & - & $96 \%$ & $77 \%$ \\
\hline Lowe $(1998)^{28}$ & $60 / 30$ & - & $92 \%$ & $90 \%$ \\
\hline Totals & $361 / 194$ & Weighted averages & $95 \%$ & $81 \%$ \\
\hline
\end{tabular}

positives are the exception. More commonly, chronic or indolent inflammatory processes which incite less intense host reactions and a lower rate of FDG accumulation are imaged. For this reason the specificity of PET in the evaluation of pulmonary opacities remains high.

False negative PET studies may also occur and are seen in three specific settings. The first pitfall occurs in tumours with relatively low metabolic activity. In a recent report four of seven bronchioalveolar tumours did not demonstrate increased FDG accumulation ${ }^{29}$ and carcinoid tumours have also had low levels of FDG accumulation in the series listed. ${ }^{23}{ }^{24}$ All other types of primary lung cancer (with variable differentiation) have increased FDG accumulation. Occasionally, well differentiated adenocarcinomas will have a relatively less marked increase in FDG accumulation, but the SUR is still abnormal. ${ }^{29}$

Size is another potential limitation for accurately identifying cancer. False negative readings can occur due to the relatively limited resolution $(5-8 \mathrm{~mm})$ of present PET tomographs and the effect that this has in detecting small abnormalities. This could play a role when the tumours are physically small or when the histological complement of malignant cells is small relative to the rest of the nodule, as might be seen in an early scar carcinoma. The Prospective Investigation of PET in Lung Nodules study ${ }^{28}$ has shown a lower sensitivity for malignancy $(80 \%)$ when the tumours were less that $1.5 \mathrm{~cm}$ in size. Although one study found no difference in the accuracy of PET when evaluating nodules $2 \mathrm{~cm}$ or less compared with those over $2 \mathrm{~cm},{ }^{27}$ this has not been universally true. This is an area where future technological advances in PET systems may improve overall accuracy. No studies have been performed to look specifically at the accuracy of PET in very small nodules although, in the studies mentioned, each had a few nodules in the $5-8 \mathrm{~mm}$ range that were characterised correctly in approximately half the cases.

The final possible, albeit uncommon, cause for false negative PET studies is hyperglycaemia. Competitive inhibition from high serum glucose levels appears to hinder FDG uptake in some cases. Research has shown that the

Table 2 Possible causes of false positive or false negative FDG PET studies

\begin{tabular}{ll}
\hline False positive FDG PET studies & False negative FDG PET studies \\
\hline Infection & Low grade malignancy \\
Acute inflammation & Microscopic or small focal malignancy \\
Recent surgical wound & Hyperglycaemia \\
Muscle hypermetabolism & Adjacent highly metabolic focus \\
\hline
\end{tabular}

inhibitory effect is most important with acute hyperglycaemia while a chronically raised glucose level only inhibits tumour uptake of FDG minimally (by about $10 \%$ ). ${ }^{8}$ Diabetic control should therefore be optimised and serum glucose values checked prior to PET imaging. If significant hyperglycaemia $(>300$ $\mathrm{mg} / \mathrm{dl}$ ) is identified, a return appointment at a time when diabetic control is improved is the suggested course of action. In the above series false negative cases from hyperglycaemia were described in only two instances.

\section{Algorithms for using PET imaging in focal lung abnormalities}

The optimal algorithm incorporating PET imaging for the evaluation of pulmonary abnormalities has not yet been identified. FDG-PET evaluation of solitary pulmonary nodules could identify metabolically inactive lesions likely to be benign. Patients with such lesions could be followed by sequential imaging studies rather than undergo invasive sampling procedures. One study has compared the use of TTNA and PET in the investigation of indeterminate nodules. The sensitivity and specificities were $100 \%$ and $78 \%$ for PET and $81 \%$ and $100 \%$ for TTNA. ${ }^{30}$ These data suggest that more malignancies may in fact be missed using a traditional TTNA approach than with PET imaging when triaging patients for thoracotomy. Conversely, a PET approach may lead to more unnecessary thoracotomies given the data from this series. One must decide whether a missed cancer diagnosis or an unnecessary thoracotomy represents the greater risk. Also of note is that nine of 33 of the patients in this series $(26 \%)$ required chest tube placement for pneumothoraces secondary to the TTNA. The costs and benefits of different diagnostic strategies are being evaluated.

\section{Staging lung cancer}

Staging of bronchogenic carcinoma is performed using the TNM system which requires accurate evaluation of the primary tumour ( $\mathrm{T})$, regional lymph nodes $(\mathrm{N})$, and distant metastasis $(M)$. The following discussion illustrates the role of FDG PET scanning for these three components of staging.

\section{PRIMARY TUMOUR}

Although tumour size can be estimated on PET imaging, thus allowing classification into $\mathrm{T} 1(<3 \mathrm{~cm})$ and $\mathrm{T} 2(>3 \mathrm{~cm})$ lesions, this is accurately determined on CT scanning. Similarly, PET scanning has poor accuracy in determining invasion into adjacent structures 
Table 3 Studies comparing PET and CT in mediastinal staging of lung cancer

\begin{tabular}{|c|c|c|c|c|c|c|c|}
\hline Reference & No. of patients & $\begin{array}{l}\text { Nodal status } \\
\text { (malignant/benign) } \\
\text { (numbers indicative of) }\end{array}$ & $\begin{array}{l}\text { Sensitivity } \\
\text { PET }\end{array}$ & $\begin{array}{l}\text { Specificity } \\
\text { PET }\end{array}$ & $\begin{array}{l}\text { Specificity } C T \text { (size } \\
\text { criteria) }\end{array}$ & Specificity $C T$ & $\begin{array}{l}\text { Statistical } \\
\text { difference PET } \\
\text { vs CT }\end{array}$ \\
\hline Chin $(1995)^{31}$ & 30 (N0-N2only) & 9/21 (patients) & $78 \%$ & $81 \%$ & $56 \%(1.5 \mathrm{~cm})$ & $86 \%$ & Not done \\
\hline Patz $(1995)^{32}$ & 42 & $23 / 39$ (stations) & $83 \%$ & $82 \%$ & $43 \%(1.0 \mathrm{~cm})$ & $85 \%$ & $\mathrm{p}<0.01$ \\
\hline Sasaki $(1996)^{33}$ & 29 & $17 / 54$ (stations) & $76 \%$ & $98 \%$ & $65 \%(1.0 \mathrm{~cm})$ & $87 \%$ & $\mathrm{p}<0.05$ \\
\hline Sazon $(1996)^{34}$ & 32 & $16 / 16$ (patients) & $100 \%$ & $100 \%$ & $81 \%(1.0 \mathrm{~cm})$ & $56 \%$ & $\mathrm{p}<0.01$ \\
\hline Scott $(1996)^{35}$ & 62 & $10 / 65$ (stations) & $100 \%$ & $98 \%$ & $60 \%(1.0 \mathrm{~cm})$ & $93 \%$ & $\mathrm{p}=0.031$ \\
\hline Steinert $(1997)^{36}$ & 47 & $58 / 133$ (stations) & $93 \%$ & $99 \%$ & $72 \%(0.7-1.1 \mathrm{~cm})$ & $94 \%$ & $\mathrm{p}=0.013$ \\
\hline Valk $(1995)^{37}$ & 74 & $24 / 52$ (sides) & $83 \%$ & $94 \%$ & $63 \%(1.0 \mathrm{~cm})$ & $73 \%$ & $\mathrm{p}<0.01$ \\
\hline Wahl $(1994)^{38}$ & 23 & $11 / 16$ (sides) & $82 \%$ & $81 \%$ & $64 \%(1.0 \mathrm{~cm})$ & $44 \%$ & $\mathrm{p}<0.05$ \\
\hline Totals & 339 & Weighted averages & $88 \%$ & $93 \%$ & $63 \%$ & $80 \%$ & \\
\hline
\end{tabular}

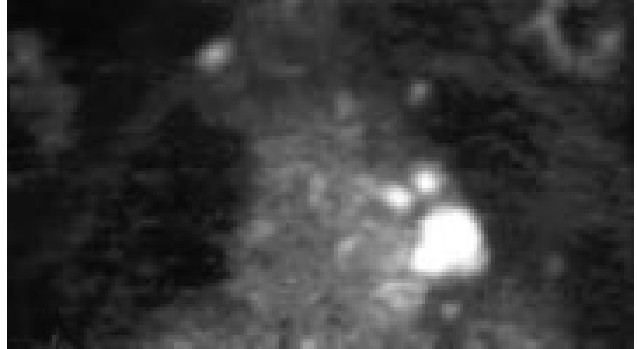

Figure 2 Coronal FDG PET image of a patient who had a chest radiograph showing a "questionable" left hilar fullness. PET imaging shows left hilar hypermetabolism associated with aorto-pulmonary nodal left lung and right supraclavicular nodal hypermetabolism, all indicative of tumour.

such as chest wall, diaphragm, spine, or large vessels-characteristics that determine $\mathrm{T} 3$ or T4 status. The identification of pleural metastasis (which constitutes T4 disease) is perhaps the most useful, if highly infrequent, discovery that can be made accurately by PET imaging when dealing with the determination of the $\mathrm{T}$ status.

REGIONAL LYMPH NODE DISEASE

PET has greater utility in nodal staging of bronchogenic carcinoma. Table 3 is a list of staging studies examining the accuracy of PET in staging lung cancer. ${ }^{31-38}$ Staging of the mediastinum can be performed by mediastinoscopy which has a sensitivity of $87-91 \%$ for disease. ${ }^{39-41}$ Staging by anatomical imaging studies such as CT scanning has been attempted but is considered by some to be only complementary to mediastinoscopy due to its poor accuracy. Adenopathy, as defined by CT imaging ( $>1 \mathrm{~cm}$ short axis diameter), is both insensitive and non-specific for malignancy. CT and MRI staging of bronchogenic carcinoma has been reported to have a sensitivity of about $50-60 \% .{ }^{42-44}$ Interestingly, nodes greater than $2 \mathrm{~cm}$ have been reported to have a $30-37 \%$ chance of being benign, ${ }^{42}{ }^{45}$ leading to the non-specificity seen for CT scanning. Figure 2 shows a case of nodal disease identified by PET imaging.

Several studies in the last few years have demonstrated the superiority of PET over CT scanning in the evaluation of nodal stage (table
3). These reports have included relatively small patient groups but the differences between PET imaging and CT scanning were statistically significant. There is minimal variation in the nodal size criteria used for CT scanning. The studies also have some variation in the rigor of nodal sampling, as noted in the number of nodal stations reported. Some of the reports only list the nodal status of individual patients while others detail the number of positive nodes. The study by Steinert and coworkers probably demonstrates the most comprehensive sampling of all of the studies. In table 3 the average sensitivity and specificity of the studies for nodal disease was $88 \%$ and $91 \%$ for PET imaging and $63 \%$ and $76 \%$ for CT scanning. This average sensitivity of PET for nodal disease is near that reported for mediastinoscopy. Of note is the lower sensitivity reported by Sasaki and coworkers ${ }^{33}$ for nodal disease. They used a PET tomograph with lower spatial resolution $(14 \mathrm{~mm})$ and may therefore have had difficulty identifying smaller tumour volumes. In fact, all four of the nodal regions that were false negative on PET imaging in their study had positive nodes that were less than $7 \mathrm{~mm}$ in size. They did identify some other nodal disease that was $7 \mathrm{~mm}$ in size.

\section{DISTANT METASTASIS}

A whole body PET image can be obtained to assess metastatic disease status. Table 4 lists three studies that looked at the data provided by PET imaging in assessing distant metastatic disease. ${ }^{37467}$ In each of the reports at least $10 \%$ of the patients were found to have distant metastasis not otherwise detected by routine chest CT scans or additional imaging studies such as bone scans. The results imply that information regarding advisability of tumour resection will be impacted by adding PET data. Not only was unsuspected disease identified on PET thus resulting in "upstaging", but many false positive findings on CT scanning (including findings outside of the chest such as adrenal nodules) were correctly interpreted as negative by PET imaging. This was illustrated in a study of 99 patients by Valk and coworkers ${ }^{37}$ in which PET correctly characterised as benign 14 of the

Table 4 FDG PET studies of lung cancer metastatic disease

\begin{tabular}{|c|c|c|c|c|c|}
\hline Reference & $\begin{array}{l}\text { No. of } \\
\text { patients }\end{array}$ & Study type & $\begin{array}{l}\text { PET detected } \\
\text { unsuspected } \\
\text { metastasis }\end{array}$ & Resectability & $\begin{array}{l}\text { Management change } \\
\text { occurred }\end{array}$ \\
\hline Bury (1996) ${ }^{46}$ & 61 & Prospective & $10 \%$ & NA & NA \\
\hline Lewis $(1994)^{47}$ & 34 & Retrospective & $29 \%$ & Changed in $18 \%$ & $41 \%$ \\
\hline Valk $(1995)^{37}$ & 99 & Prospective & $11 \%$ & NA & NA \\
\hline
\end{tabular}



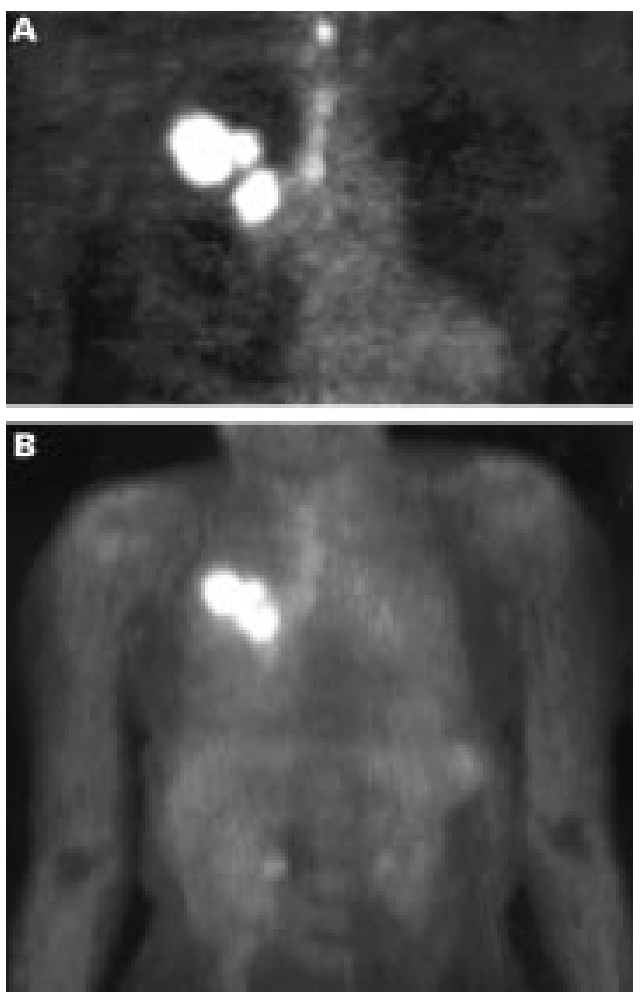

Figure 3 (A) Coronal PET image of a 61 year old woman with a large right lung mass (non-small cell tumour) on CT scan with associated right hilar $(2 \mathrm{~cm})$ and right paratracheal adenopathy $(1 \mathrm{~cm})$ showing

hypermetabolism in these regions. CT scanning also showed multiple hypodense liver lesions, the largest of which was 4 $\mathrm{cm}$, which were described as cysts or metastases. (B) Coronal PET body image of the same patient showing no evidence of liver metastasis.

19 false positive findings reported on CT scans. Thus, PET imaging could aid in identifying cancer patients as surgical candidates who might otherwise be considered unresectable. In considering both contributions, the change in management brought about by the PET information can reach very high proportions of study populations as is illustrated by Lewis and coworkers ${ }^{47}$ where management changes occurred in $41 \%$ of patients. Figure 3 is an example of PET scan findings that characterised other imaging abnormalities as benign.

The adrenal glands are commonly a dilemma in the examination of metastatic disease. Benign enlargement of the adrenal glands occurs commonly and is difficult to dis-

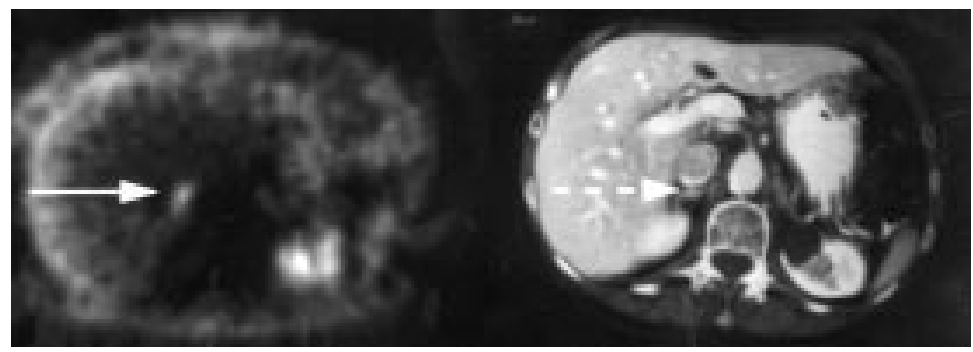

Figure 4 Axial abdominal PET and CT images of a patient with right lung cancer. The $C T$ scans of the chest and abdomen were described as normal, other than the right lung mass. PET imaging showed hypermetabolism indicative of tumour in the right lung but, in addition, demonstrated right adrenal disease (arrow). Needle biopsy specimens showed tumour in the right adrenal gland (dashed arrow), changing the patient from resectable to unresectable. tinguish from metastatic enlargement of the adrenal glands in patients with lung cancer. Unenhanced CT scans can produce a sensitivity and specificity of about $96 \%$ and $73 \%$ for metastatic disease to enlarged adrenal glands. ${ }^{48}$ However, this would commonly necessitate a second adrenal imaging session if CT scanning is performed with contrast medium. MRI of the adrenals using chemical shift imaging can distinguish benign from malignant enlarged adrenal glands with nearly $100 \%$ accuracy according to some reports. ${ }^{49}$ However, this would also require a separate imaging procedure. Furthermore, evaluation by these methods is predicated on anatomical enlargement and normal adrenal glands with tumour may not be detected. It should also be considered that the accuracy of adrenal size characterisation by CT scanning is only good to about \pm 1 $\mathrm{cm}^{50}$ Percutaneous biopsy specimens are usually employed to evaluate the possibility of lung cancer metastasis to an enlarged suspicious adrenal gland. However, percutaneous biopsy itself only has an accuracy of about $90 \%$ for metastatic disease. ${ }^{48}$

PET imaging has been shown to have the ability to distinguish benign adrenal enlargement from metastatic disease. In one study of 20 patients by Boland et $a \bar{F}^{1}$ PET imaging was able to show a statistically significant difference in lesion metabolism between benign enlarged adrenal glands and those with malignancy. Maurea and colleagues ${ }^{52}$ found similar results in their study of 15 patients with enlarged adrenal glands. Interestingly, in this study there was no statistically significant difference between the size of the adrenal glands in the benign group compared with the group with malignant enlargement. Recently a study by Erasmus $^{53}$ showed that, in 27 patients (33 enlarged adrenal glands) with bronchogenic carcinoma, PET imaging correctly identified all adrenal glands with tumour. Eight of 10 benign enlarged adrenal glands had no increase in FDG accumulation and were therefore appropriately identified as being benign by PET. The advantage of using PET imaging in this setting is that, unlike the other imaging modalities, the information about the adrenal glands is obtained in the same imaging session. Also, the technique has the ability to identify tumour in adrenal glands that are normal in size (fig 4). However, the accuracy of PET imaging in assessing normal adrenal glands for metastasis has not been documented.

COST/BENEFIT OF PET STAGING OF LUNG CANCER Gambhir and coworkers developed a cost/ benefit model that demonstrated a significant cost savings by including FDG-PET imaging in the staging of lung cancer. ${ }^{44}$ They found that a conservative strategy of using chest CT scanning plus PET imaging showed a potential cost savings of $\$ 1154$ per patient without a loss of life expectancy. This strategy would involve taking biopsy specimens from all positive findings with either CT or PET imaging that might indicate non-resectable malignancy so that $100 \%$ of surgical candidates are identified definitively. Chest CT scanning is performed in 


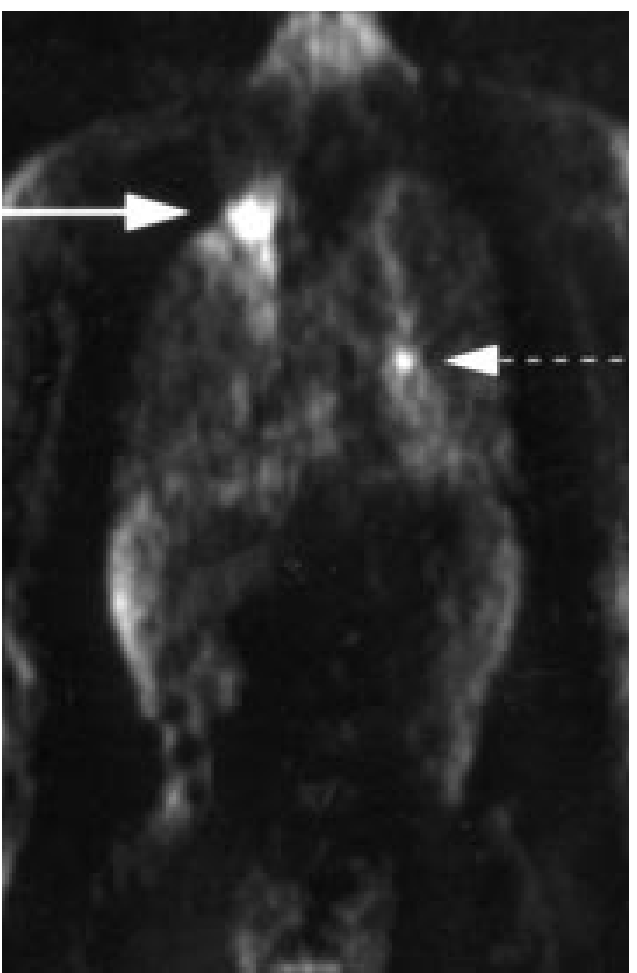

Figure 5 Coronal FDG PET image of a patient with a history of a right upper lobe lung cancer treated with radiation and chemotherapy two years prior to imaging who presented with a new nodular abnormality with pleural thickening in the right apex on CT scanning. The PET scan shows hypermetabolism (solid arrow) that is indicative of tumour recurrence which was confirmed by biopsy. A left hilar focus of hypermetabolism (broken arrow) is also suspicious for disease but was not confirmed.

all patients to insure that proper anatomical information is available to the surgeon. A less conservative strategy in which patients with concordant positive results on CT and PET scanning indicating non-resectability do not undergo biopsy or proceed to thoracotomy would result in a cost saving of $\$ 2267$ but would miss $1.7 \%$ of potentially operable patients. The largest component of the cost savings realised by these approaches results from the prevention of unnecessary surgery and biopsy procedures.

\section{Assessing lung cancer therapy and recurrence}

Patients with bronchogenic carcinoma commonly present in an advanced stage and, as such, have a five year survival of $13 \% .^{54}$ An accurate assessment of the impact of chemotherapy and radiation therapy would be of enormous benefit in directing treatment for patients with advanced stages of lung cancer. Historically clinicians have used tumour shrinkage to assess efficacy, but this may not be the best indicator of response to treatment. FDG-PET imaging can identify changes in

Table 5 FDG PET studies of recurrent lung cancer

\begin{tabular}{llll}
\hline Reference & $\begin{array}{l}\text { Total no. of patients } \\
\text { (malignant/ benign) }\end{array}$ & Sensitivity PET & Specificity PET \\
\hline Patz $(1995)^{58}$ & $43(35 / 8)$ & $97 \%$ & $100 \%$ \\
Inoue $(1995)^{59}$ & $38(23 / 13)$ & $94 \%$ & $89 \%$ \\
\hline
\end{tabular}

glucose uptake and may prove to be a better indicator of a favourable response to treatment.

It may be important to differentiate between a decrease in FDG uptake compared with the complete absence of FDG uptake. Some investigators have concluded that a decrease in FDG uptake does not necessarily indicate a good prognosis. ${ }^{55}$ Rather, it has been suggested that a decrease in FDG uptake may only indicate a partial response due to destruction of cells sensitive to the therapy while other resistant cells continue to be metabolically active. Normalisation of the FDG uptake after treatment, on the other hand, appears to be a good prognostic sign. One study by Hebert and coworkers ${ }^{56}$ demonstrated that negative PET findings after radiation therapy, even in the presence of nonspecific radiographic changes, are an indicator of a good response. Hebert noted that all of their patients with negative PET findings were alive at two years after treatment whereas $50 \%$ of patients with residual hypermetabolism, albeit reduced, had died within that same two year period. Other investigators have used this logic to justify further treatment of asymptomatic individuals whose PET scans demonstrate residual hypermetabolism after an initial course of therapy. Frank and colleagues treated five such asymptomatic patients in their study based solely on residual hypermetabolism and all were alive at three years. ${ }^{57}$

Early diagnosis of recurrent lung cancer is another potential use of FDG-PET imaging. Radiological changes such as scarring and necrosis which occur after treatment may obscure the identification of recurrent tumour unless significant volume changes occur over time. The interpretation of recurrence is often not made until the disease progresses to the point of marked enlargement of questionable abnormalities. Unfortunately, a tissue biopsy specimen that is negative for tumour in such situations is suspect due to the inherent difficulty in identifying and accurately sampling the areas of viable tumour hidden in the midst of the scar. A PET evaluation of tumour recurrence can potentially assist in this determination. Patients who have chest radiographic findings suspicious for tumour recurrence can be accurately characterised by FDG-PET imaging as demonstrated in fig 5 .

Benign, non-specific pleural thickening is another example of post-treatment changes which may be difficult to differentiate from recurrent disease. Pleural biopsy itself may be relatively unreliable when performed percutaneously. PET imaging will probably differentiate recurrent tumour from radiation inducing benign pleural thickening. Several investigators have examined the use of PET imaging for just these purposes (table 5). Patz and coworkers ${ }^{58}$ demonstrated a very high accuracy of PET imaging in distinguishing recurrent disease from benign treatment effects when patients were scanned 12-24 months after therapy. The report of Inoue and coworkers ${ }^{59}$ yielded similar results.

There are potential pitfalls for FDG-PET imaging when used for this purpose. Occasionally treatment can induce hypermetabolic 


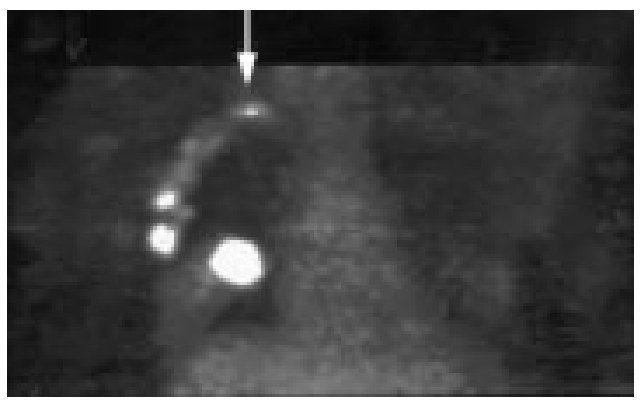

Figure 6 Coronal FDG PET image of a patient with right breast cancer and multiple positive lymph nodes including infraclavicular lymph nodes (arrow) that were confirmed on extensive axillary dissection.

inflammatory changes that may make it difficult to differentiate persistent tumour from treatment effect. This is most common when radiation therapy has been used. Chemotherapy does not commonly induce the tissue damage that leads to inflammatory hypermetabolism. The likelihood of seeing moderate levels of hypermetabolism after radiation therapy diminishes as time passes. Scans are likely to be most reliable when a year or more has passed from the last radiation treatment. The most common finding indicative of post-therapy inflammatory hypermetabolism is diffuse, mildly raised FDG accumulation in the soft tissues of radiation port regions. ${ }^{58} \mathrm{~A}$ cutoff of 2.5 for the SUR still appears to be accurate in differentiating tumour from benign changes in focal abnormalities identified at the posttreatment setting.

\section{Evolving applications of PET in thoracic oncology}

PET imaging may play a role in evaluating other kinds of thoracic cancer. Data are available that have shown the usefulness of PET imaging in identifying breast cancer and staging axillary lymph nodes. ${ }^{6061}$ Data from one investigator have shown that PET staging of the axilla detected all of the nodal disease surgically confirmed in a group of 124 patients. ${ }^{62}$ Figure 6 shows a case of nodal disease accurately characterised by PET in a patient with breast cancer.

Preliminary reports have shown that FDGPET may help in the evaluation of mediastinal tumours. Thymomas have demonstrated variable FDG uptake which appears to correlate with their invasiveness. Benign cysts and thymic hyperplasia have low FDG accumulation. ${ }^{6364}$ Moderate levels of FDG accumulation have been seen in myeloma and schwannoma, whereas low uptake has been observed in teratoma and various benign cysts. $^{64}$ Mediastinal lymphomas have high FDG accumulation and can be accurately identified using PET imaging. ${ }^{65}$ Increased FDG uptake in lymphomas correlates with high histological grade of malignancy and a high proliferation rate. ${ }^{66}$ There are also data to indicate the ability of PET imaging to stage lymphoma with improved accuracy over CT scanning. ${ }^{65}$ Others have shown that FDG-PET imaging may have greater sensitivity than gallium in evaluating lymphomas. ${ }^{67}$
There has been some work done in the evaluation of chest wall sarcomas. FDG accumulation correlates with sarcoma grade and, although high grade lesions can be distinguished from benign lesions, low grade neoplasms may appear similar to benign lesions. $^{68-70}$ A known high grade lesion may therefore be amenable to PET imaging for determining stage or evaluating disease recurrence after treatment.

Although a great deal of data has been accumulated regarding PET evaluation of solitary pulmonary masses, little specific information exists on the usefulness of PET in evaluating multiple pulmonary masses. Nevertheless, reports have described the accuracy of FDGPET as being greater than other imaging modalities in identifying pulmonary metastasis from other malignancies such as colon cancer ${ }^{71}$ or melanoma. ${ }^{72} 73$

\section{Evaluation of oesophageal malignancy}

Oesophageal cancer will be diagnosed in 11300 people in the USA this year and is considered to be one of the most important cancers of developing countries. ${ }^{74}$ The incidence of the disease is much higher in Asia, Northern France, and some other scattered regions in which oesophageal cancer is endemic. The overall survival rate is $10 \%$ or less at five years because of the advanced stage of disease at the time of presentation.

Early stage oesophageal cancer is often asymptomatic and detection is uncommon prior to advanced stages of tumour spread. Unfortunately, current staging techniques are still inaccurate and understage oesophageal malignancies in a significant portion of patients. More precise staging will be critical for treatment of patients with oesophageal cancer and could allow truly "early stage" patients to undergo immediate surgery while advanced stage patients would be referred for the promising multimodality treatment protocols. Current routine staging studies are not acceptably sensitive.

Endoscopic evaluation is an accepted method of evaluating oesophageal cancer and has the advantages of allowing both direct visualisation and tissue sampling. Although the addition of endoscopic ultrasound has added an important tool for the detection of paraoesophageal lymph nodes, the accuracy of this technique is operator dependent and somewhat controversial. It certainly has no role in the detection of distant disease.

Non-invasive imaging with chest radiography and chest and abdomen CT scanning is performed to identify lesions in the liver, adrenal glands or lung but is notoriously inaccurate in identifying non-bulky lymphadenopathy and has no hope of spotting lesions that are more distant. New data are emerging regarding the use of PET in staging oesophageal cancer. Several centres have reported substantial advantages to staging oesophageal cancer with PET imaging when compared with conventional techniques. In one study PET had a sensitivity for predicting local nodal disease of $76 \%$ (22/29) compared with $45 \%$ (13/29) for CT 

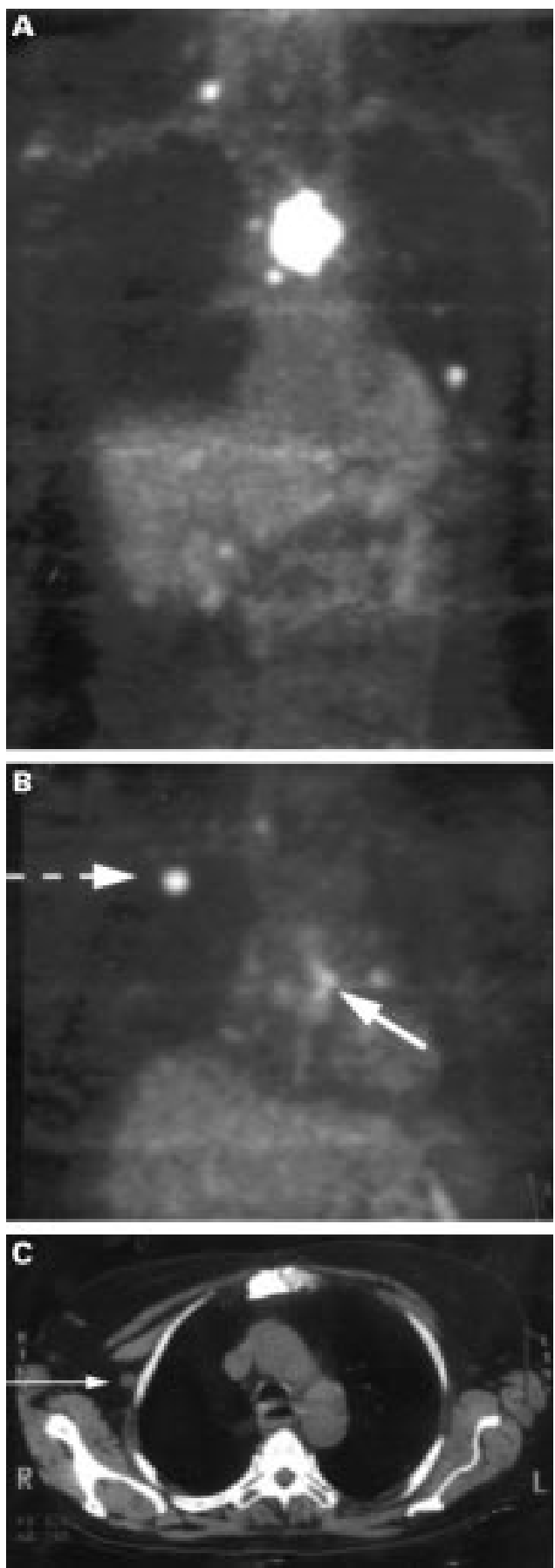

Figure 7 (A) Coronal FDG PET image of a patient showing a large upper oesophageal tumour with a left lung metastasis, local nodal disease, and a right supraclavicular nodal metastasis. CT scanning of the head and neck, chest and abdomen described the oesophageal tumour and a $1 \mathrm{~cm}$ left lower lobe nodule only. (B) Coronal FDG PET image of a patient showing a small mid oesophageal tumour (arrow) with local nodal disease and a right axillary nodal metastatsis (dashed arrow). (C) After an initial negative $C T$ scan of the chest a follow up $5 \mathrm{~mm}$ slice $C T$ scan of the chest showed a $1 \mathrm{~cm}$ right axillary lymph node (arrow) not identified on the initial CT scan.

scanning ${ }^{75}$ in patients who underwent curative surgery. In the same study, in another seven patients who did not undergo surgery PET detected distant metastases in five patients who had no metastatic disease identified on CT scanning. Inappropriate surgery in these patients was thereby prevented by the PET find- ings. Another patient had an unsuspected concomitant lung primary discovered with PET imaging. In another study of 35 patients with potentially resectable oesophageal cancer, as determined by CT scanning, distant metastatic disease was identified by PET imaging in $20 \%$. The accuracy of PET for determining distant metastatic disease in this group was $91 \%{ }^{76}$ Figure 7 illustrates patients with oesophageal cancer in whom distant disease was identified only with PET scans and subsequently confirmed surgically, thus averting surgical resection. The use of PET imaging in patients with oesophageal cancer could lead to more accurate staging and allow more accurate stratification of patients into surgical and multimodality protocols. Further work will need to be done to see if the introduction of PET imaging into the staging work up of these patients will lead to any survival benefit. Ultimately any substantial improvement in the survival rates will depend upon successful treatment regimens. In the short term some operative risk may be avoided in patients with previously undocumented metastatic disease.

\section{Summary}

Continuing advances in PET imaging have resulted in an improved ability to evaluate thoracic malignancies. Published reports demonstrate that PET provides accurate, non-invasive detection and staging of thoracic malignancy. Preliminary studies suggest that PET may also be able to assess the therapeutic response accurately. The studies investigating PET have been relatively small but have shown statistically significant advantages over conventional non-invasive techniques in accuracy and possibly even cost/benefit performance in thoracic malignancies.

1 Warburg O. The metabolism of tumors. New York: Richard R Smith, 1931: 129-69.

2 Weber G. Enzymology of cancer cells. Part I. N Engl f Med 1977;296:468-92.

3 Weber G. Enzymology of cancer cells. Part II. N Engl f Med 1977;296:541-51

4 Nolop KB, Rhodes CG, Brudin LH, et al. Glucose utilization in vivo by human pulmonary neoplasms. Cancer 1987;60:2682-9.

5 Gallagher BM, Fowler JS, Gutterson NI, et al. Metabolic trapping as a principle of radiopharmaceutical design: some factors resposible for the biodistribution of $\left({ }^{18} \mathrm{~F}\right)$ 2-deoxy-2-fluoro-D-glucose. $7 \mathrm{Nucl}$ Med 1978;19:1154-61.

6 Duhaylongsod FG, Lowe VJ, Patz EJ, et al. Lung tumor growth correlates with glucose metabolism measured by fluoride-18 fluorodeoxyglucose positron emission tomography. Ann Thorac Surg 1995;60:1348-52.

7 Langen KJ, Braun U, Rota KE, et al. The influence of plasma glucose levels on fluorine-18-fluorodeoxyglucose uptake in bronchial carcinomas. F Nucl Med 1993;34:3559 .

8 Torizuka T, Clavo AC, Wahl RL. Effect of hyperglycemia on in vitro tumor uptake of tritiated FDG, thymidin L-methionine and L-leucine. $\mathcal{F} \mathrm{Nucl}$ Med 1997;38:382-6.

9 Lowe VJ, DeLong DM, Hoffman JM, et al. Optimum scanning protocol for FDG-PET evaluation of pulmonary malignancy. F Nucl Med 1995;36:883-7.

10 Gurney JW. Determining the likelihood of malignancy in solitary pulmonary nodules with Bayesian analysis. Part I. Theory. Radiology 1993;186:405-13.

11 Gurney JW, Lyddon DM, McKay JA. Determining the likelihood of malignancy in solitary pulmonary nodules with Bayesian analysis. Part II. Application. Radiology 1993;186: 415-22.

12 Schenk DA, Bryan CL, Bower JH, et al. Transbronchial needle aspiration in the diagnosis of bronchogenic carcinoma. Chest 1987;92:83-5.

13 Salathe M, Soler M, Bolliger CT, et al. Transbronchial needle aspiration in routine fiberoptic bronchoscopy. Respiration $1992 \cdot 59 \cdot 5-8$

14 Wang KP, Kelly SJ, Britt JE. Percutaneous needle aspiration biopsy of chest lesions. New instrument and new technique. Chest 1988;93:993-7. 
15 Polak J, Kubik A. Percutaneous thin needle biopsy of malignant and nonmalignant thoracic lesions. Radiol Diagn nant and nonm $1989 ; 30: 177-82$.

16 Winning AJ, McIvor J, Seed WA, et al. Interpretation of negative results in fine needle aspiration of discrete pulmonary lesions. Thorax 1986;41:875-9.

17 Daly BD, Faling LJ, Diehl JT, et al. Computed tomographyguided minithoracotomy for the resection of small peripheral pulmonary nodules. Ann Thorac Surg 1991;51:465-9.

18 Williams AJ, Santiago S, Lehrman S, et al. Transcutaneous needle aspiration of solitary pulmonary masses: how many passes? Am Rev Respir Dis 1987;136:452-4.

19 Mack MJ, Hazelrigg SR, Landreneau RJ, et al. Thoracoscopy for the diagnosis of the indeterminate solitary scopy for the diagnosis of the indeterminate solit
pulmonary nodule. Ann Thor Surg 1993;56:825-30.

20 Dewan NA, Gupta NC, Redepenning LS, et al. Diagnostic efficacy of PET-FDG imaging in solitary pulmonary nodules. Potential role in evaluation and management Chest 1993;104:997-1002.

21 Patz EJ, Lowe VJ, Hoffman JM, et al. Focal pulmonary abnormalities: evaluation with F-18 fluorodeoxyglucose PET scanning. Radiology 1993;188:487-90.

22 Bury T, Dowlati A, Paulus P, et al. Evaluation of the solitary pulmonary nodule by positron emission tomography imaging. Eur Respir f 1996;9:410-4.

23 Duhaylongsod FG, Lowe VJ, Patz EJ, et al. Detection of primary and recurrent lung cancer by means of F-18 mary and recurrent lung cancer by means of F-18 fluorodeoxyglucose positron emission tomograph
PET). F Thorac Cardiovasc Surg 1995;110:130-9.

24 Gupta NC, Maloof J, Gunel E. Probability of malignancy in solitary pulmonary nodules using fluorine-18-FDG and PET (see comments). F Nucl Med 1996;37:943-8.

25 Knight SB, Delbeke D, Stewart JR, et al. Evaluation of pulmonary lesions with FDG-PET. Comparison of findings in patients with and without a history of prior malignancy. Chest 1996;109:982-8.

26 Kubota K, Matsuzawa T, Fujiwara T, et al. Differential diagnosis of lung tumor with positron emission tomography: a prospective study. F Nucl Med 1990;31:1927-32.

27 Lowe VJ, Duhaylongsod FG, Patz EF, et al. Pulmonary abnormalities and PET data analysis: a retrospective study. abnormalities and PET da
Radiology 1997;202:435-9.

28 Lowe VJ, Fletcher J W, Gobar L, et al. Prospective investigation of PET in lung nodules. $\mathcal{F}$ Clin Oncol 1998;16:107584.

29 Higashi K, Seki H, Taniguchi M, et al. Bronchioloalveolar carcinoma: false-negative results on FDG-PET. $f$ Nucl Med 1997;38:79P.

30 Dewan NA, Reeb SD, Gupta NC, et al. PET-FDG imaging and transthoracic needle lung aspiration biopsy in evaluation of pulmonary lesions. A comparative risk-benefit analysis. Chest 1995;108:441-6.

31 Chin RJ, Ward R, Keyes JW, et al. Mediastinal staging of non-small-cell lung cancer with positron emission tomonon-small-cell lung cancer with positron emission
graphy. Am $\mathcal{F}$ Respir Crit Care Med 1995;152:2090-6.

32 Patz EJ, Lowe VJ, Goodman PC, et al. Thoracic nodal staging with PET imaging with 18 FDG in patients with bronchogenic carcinoma. Chest 1995;108:1617-21.

33 Sasaki M, Ichiya Y, Kuwabara Y, et al. The usefulness of FDG positron emission tomography for the detection of mediastinal lymph node metastases in patients with non-small cell lung cancer: a comparative study with X-ray computed tomography. Eur F Nucl Med 1996;23:741-7.

34 Sazon DA, Santiago SM, Soo HG, et al. Fluorodeoxyglucose-positron emission tomography in the detection and staging of lung cancer. Am $\mathcal{f}$ Respir Crit Care Med 1996;153:417-21.

35 Scott WJ, Gobar LS, Terry JD, et al. Mediastinal lymph node staging of non-small-cell lung cancer: a prospective comparison of computed tomography and positron emission tomography. $\mathcal{F}$ Thorac Cardiovasc Surg 1996;111:6428.

36 Steinert HC, Hauser M, Allemann F, et al. Non-small cell lung cancer: nodal staging with FDG PET versus CT with correlative lymph node mapping and sampling. Radiology 1997;202:441-6.

37 Valk PE, Pounds TR, Hopkins DM, et al. Staging non-small cell lung cancer by whole-body positron emission tomographic imaging. Ann Thorac Surg 1995;60:1573-81.

38 Wahl RL, Quint LE, Greenough RL, et al. Staging of mediastinal non-small cell lung cancer with FDG PET, CT, and fusion images: preliminary prospective evaluation. Radiology 1994;191:371-7.

39 Gdeedo A, Van SP, Corthouts B, et al. Prospective evaluation of computed tomography and mediastinoscopy in mediastinal lymph node staging. Eur Respir f 1997;10 1547-51

40 Patterson GA, Ginsberg RJ, Poon PY, et al. A prospective evaluation of magnetic resonance imaging, computed tomography, and mediastinoscopy in the preoperative assessment of mediastinal node status in bronchogenic carcinoma. $\mathcal{F}$ Thorac Cardiovasc Surg 1987;94:679-84.

41 Van Schil PE, Van HRH, Schoofs EL. The value of mediastinoscopy in preoperative staging of bronchogenic carcinoma. F Thorac Cardiovasc Surg 1989;97:240-4.

42 McLoud TC, Bourgouin PM, Greenberg RW, et al. Bronchogenic carcinoma: analysis of staging in the mediastinum with CT by correlative lymph node mapping and sampling. Radiology 1992;182:319-23.

43 Webb WR, Gatsonis C, Zerhouni EA, et al. CT and MR imaging in staging non-small cell bronchogenic carcinoma: report of the Radiologic Diagnostic Oncology Group. Radiology 1991;178:705-13.
44 Wittens $\mathrm{CH}$, Bollen EC, van DC, et al. Accuracy of computed tomography of the mediastinum in bronchocomputed tomography of the mediastinum in bro

45 Whittlesey D. Prospective computed tomographic scanning in the staging of bronchogenic cancer. $\mathcal{F}$ Thorac Cardiovasc Surg 1988;95:876-82.

46 Bury T, Dowlati A, Paulus P, et al. Staging of non-small-cell ung cancer by whole-body fluorine-18 deoxyglucose positron emission tomography. Eur 7 Nucl Med 1996;23:204-6.

47 Lewis P, Griffin S, Marsden P, et al. Whole-body $18 \mathrm{~F}$-fluorodeoxyglucose positron emission tomography in preoperative

48 Korobkin M, Brodeur FJ, Yutzy GG, et al. Differentiation of adrenal adenomas from nonadenomas using CT attenuation values. $A 7 R$ 1996;166:531-6.

49 Schwartz LH, Panicek DM, Doyle MV, et al. Comparison of two algorithms and their associated charges when evaluating adrenal masses in patients with malignancies. AfR ing adrenal masses

50 Linos DA, Stylopoulos N. How accurate is computed tomography in predicting the real size of adrenal tumors? A ;32:740-3.

51 Boland GW, Goldberg MA, Lee MJ, et al. Indeterminate adrenal mass in patients with cancer: evaluation at PET with 2-[F-18]-fluoro-2-deoxy-D-glucose. Radiology 1995; 194:131-4.

52 Maurea S, Mainolfi $\mathrm{C}$, Wang $\mathrm{H}$, et al. Positron emission tomography (PET) with fludeoxyglucose F-18 in the study of adrenal masses: comparison of

53 Erasmus JJ, Patz EJ, McAdams HP, et al. Evaluation of adrenal masses in patients with bronchogenic carcinoma using $18 \mathrm{~F}$-fluorodeoxyglucose positron emission tomography (see comments). AfR 1997;168:1357-60.

54 Recine D, Rowland K, Reddy S, et al. Combined modality therapy for locally advanced non-small cell lung carcinoma. Cancer 1990;66:2270-8.

55 Ichiya Y, Kuwabara Y, Otsuka M, et al. Assessment of response to cancer therapy using fluorine-18fuorodeoxyglucose and positron emission tomography. $\mathcal{F}$ Nucl Med 1991;32:1655-60.

56 Hebert ME, Lowe VJ, Hoffman JM, et al. Positron emission tomography in the pretreatment evaluation and follow-up of non-small cell lung cancer patients treated with radiotherapy: preliminary findings. $\mathrm{Am} \mathcal{f} \mathrm{Clin}$ Oncol 1996;19:416-21.

57 Frank A, Lefkowitz D, Jaeger S, et al. Decision logic for retreatment of asymptomatic lung cancer recurrence based on positron emission tomography findings. Int $\mathcal{F}$ Radiat Oncol Biol Phys 1995;32:1495-512.

58 Patz EJ, Lowe VJ, Hoffman JM, et al. Persistent or recurrent bronchogenic carcinoma: detection with PET and 2-[F18]-2-deoxy-D-glucose. Radiology 1994;191:379-82.

59 Inoue T, Kim EE, Komaki R, et al. Detecting recurrent or residual lung cancer with FDG-PET. F Nucl Med 1995;36: 788-93.

60 Adler LP, Crowe JP, al KN, et al. Evaluation of breast masses and axillary lymph nodes with [F-18] 2-deoxy-2-fluoro-Dglucose PET. Radiology 1993;187:743-50.

61 Avril N, Dose J, Janicke F, et al. Assessment of axillary lymph node involvement in breast cancer patients with positron emission tomography using radiolabeled 2-(fluorine-18)-fluoro-2-deoxy-D-glucose. $f$ Natl Cancer Inst 1996;88:1204-9.

62 Utech CI, Young CS, Winter PF. Prospective evaluation of fluorine-18 fluorodeoxyclucose positron emission tomography in breast cancer for staging of the axilla related to surgery and immunocytochemistry. Eur $7 \mathrm{Nucl}$ Med $6 ; 23: 1588-93$

63 Liu RS, Yeh SH, Huang MH, et al. Use of fluorine-18 fluorodeoxyglucose positron emission tomography in the
detection of thymoma: a preliminary report. Eur $\mathcal{f} \mathrm{Nucl}$ Med 1995;22:1402-7.

64 Kubota K, Yamada S, Kondo T, et al. PET imaging of primary mediastinal tumours. Br f Cancer 1996;73:882-6.

65 Newman JS, Francis IR, Kaminski MS, et al. Imaging of lymphoma with PET with 2-[F-18]-fluoro-2-deoxy-Dglucose: correlation with CT. Radiology 1994;190:111-6.

66 Lapela M, Leskinen S, Minn HR, et al. Increased glucose metabolism in untreated non-Hodgkin's lymphoma: a study with positron emission tomography and fluorine-18fluorodeoxyglucose. Blood 1995;86:3522-7.

67 Paul R. Comparison of fluorine-18-2-fluorodeoxyglucose and gallium-67 citrate imaging for detection of lymphoma. 7 Nucl Med 1987;28:288-92.

68 Garcia R, Kim EE, Wong FC, et al. Comparison of fluorine18-FDG PET and technetium-99m-MIBI SPECT in evaluation of musculoskeletal sarcomas. F Nucl Med 1996; 37:1476-9.

69 Kole AC, Nieweg OE, van GR, et al. Detection of local recurrence of soft-tissue sarcoma with positron emission tomography using [18F]fluorodeoxyglucose. Ann Surg Oncol 1997;4:57-63.

70 Nieweg OE, Pruim J, van GR, et al. Fluorine-18fuorodeoxyglucose PET imaging of soft-tissue sarcoma. $f$ Nucl Med 1996;37:257-61.

71 Beets G, Penninckx F, Schiepers C, et al. Clinical value of whole-body positron emission tomography with $[18 \mathrm{~F}]$ fluorodeoxyglucose in recurrent colorectal cancer. $\mathrm{Br}$ f Surg 1994;81:1666-70.

72 Boni R. Whole-body positron emission tomography: an accurate staging modality for metastatic melanoma. Arch Dermatol 1996;132:833-4. 
73 Damian DL, Fulham MJ, Thompson E, et al. Positron emission tomography in the detection and management of emission tomography in the detection and managem
metastatic melanoma. Melanoma Res 1996;6:325-9.

74 Parkin DM, Pisani P, Ferlay J. Estimates of the worldwide incidence of eighteen major cancers in 1985. Int $\mathcal{F}$ Cancer 1993;54:594-606.
75 Flanagan FL, Dehdashti F, Siegel BA, et al. Staging of esophageal cancer with $18 \mathrm{~F}$-fluorodeoxyglucose positron emission tomography. $A \not R$ 1997;168:417-24.

76 Luketich JD, Schauer PR, Meltzer CC, et al. Role of positron emission tomography in staging esophageal cancer. Ann Thorac Surg 1997;64:765-9. 